\section{CPS-087 EVOLUTION OVER TIME OF ANTIRETROVIRALS}

${ }^{1} S$ Hernandez Rojas*, ${ }^{1}$ MDP Díaz Ruiz, ${ }^{1}$ JA de León Gil, ${ }^{2}$ M Miguelez Morales, ${ }^{1}$ M Suarez Gonzalez, ${ }^{2}$ A Estévez López, '1 Merino Alonso, 'E Ramos Santana, ${ }^{1} \mathrm{~J} A$ Martín Conde. ${ }^{1}$ Hospital Nuestra Señora de la Candelaria, Hospital Pharmacy, Santa Cruz de Tenerife, Spain; ${ }^{2}$ Hospital Nuestra Señora de la Candelaria, Internal Medicine, Santa Cruz de Tenerife, Spain

10.1136/ejhpharm-2019-eahpconf.236

Background GESIDA 2018, the national guideline of AIDS in Spain, recommends, in the initial antiretroviral treatment (HAART) in adults infected with HIV, to use a simple target regime (STR) to favour adherence.

Purpose To analyse how naive HIV treatment has changed over the years both in economic aspects and in usual clinical practice.

Material and methods Longitudinal and descriptive observational study of the series of cases that began during the period January 2013 - September 2018 with antiretrovirals, naive patients only.The data have been extracted from the electronic medical record through the DRAGOAE and Farmatools program, are collected in an Excel spreadsheet and descriptive statistics are then made. The main variables under study were: initial antiretroviral treatment, patient cost per year and date of treatment initiation.

Results During this study period, 841 naive patients started treatment.

The patients who started per year from 2013 to 2017 were: 105, 180, 140, 146 and 170, respectively. The total annual cost of HAART was: $€ 767,966.83, € 1,339,510.52$, $€ 1,017,660.24$, $€ 1,051,474.85$ and $€ 1,122,105,72$, respectively.

From January to September 2018, 100 patients started antiretroviral treatment and the total cost has been $€ 584,434.94$. The average cost per patient and year from 2013 to 2018 was: $€ 7,313.96, € 7,441.72, \quad € 7,269, \quad € 7,201.88$, $€ 6,600.62$ and $€ 5,844.35$, respectively.

The treatments most frequently prescribed in the years 2013 to 2018 were:

- 2013: emtricitabine/tenofovir-disoproxil/rilpivirine (FTC/ TDF/RPV) (31\%), emtricitabine/tenofovir-disoproxil/efavirenz (FTC/TDF/EFV) (24\%) and darunavir/ritonavir (DRV/r) + FTC/TDF (23\%).

- 2014: FTC/TDF/RPV (30\%), DRV/r+FTC/TDF (25\%) and FTC/TDF/EFV (11\%).

- 2015: abacavir/lamivudine/dolutegravir (ABC/3TC/DLG) (23\%), FTC/TDF/RPV (16\%), emtricitabine/tenofovirdisoproxil/elvitegravir/cobicistat (FTC/TDF/EVG/COBI) (13\%) and FTC/TDF+DLG (13\%).

- 2016: ABC/3TC/DLG (30\%), FTC/TDF/EVG/COBI (19\%) and FTC/TDF+DLG (16\%).

- 2017: ABC/3TC/DLG (29\%), emtricitabine/tenofoviralafenamide/elvitegravir/cobicistat (FTC/TAF/EVG/COBI) (27\%) and FTC/TDF+DLG (19\%).

- 2018: ABC/3TC/DLG (28\%), FTC/TDF+DLG (26\%) and FTC/TAF/EVG/COBI (20\%).

Conclusion The integrase inhibitors (DLG and EVG) have become the third drug of choice in the HAART of the naive patients. Moving to IPs and NNRTIs, the combination ABC/ DLG/3TC is the most prescribed since 2015, and it is the most cost-effective STR in Spain. The average cost per patient has decreased by $€ 1500$ year on average compared to 2014. This is due to the high use of $\mathrm{ABC} / \mathrm{DLG} / 3 \mathrm{TC}$ and the current low cost of the combination FTC/TDF.
REFERENCES AND/OR ACKNOWLEDGEMENTS

To my service.

No conflict of interest.

\section{CPS-088 EFFECTIVENESS OF GLECAPREVIR/PIBRENTASVIR FOR THE TREATMENT OF CHRONIC HEPATITIS C}

${ }^{1} \mathrm{C}$ Burgui, ${ }^{2} \mathrm{R}$ Juanbeltz, ${ }^{2} \mathrm{~J}$ Castilla, ${ }^{3} \mathrm{~B}$ Larrayoz ${ }^{*},{ }^{3} \mathrm{M}$ Sarobe, ${ }^{4} \mathrm{JM}$ Zozaya, ${ }^{5} \mathrm{M}$ Gracia-Ruiz de Alda, ${ }^{3} \mathrm{R}$ San Miguel. 'Ciber Epidemiologia y Salud Publica Ciberesp, Hospital Pharmacy, Pamplona, Spain; ${ }^{2}$ Instituto de Salud Publica de Navarra-Idisna- Ciber Epidemiología y Salud Pública Ciberesp, Transmissible Disease and Vaccination, Pamplona, Spain; ${ }^{3}$ Navarra Hospital Complex-Idisna, Hospital Pharmacy, Pamplona, Spain; ${ }^{4}$ Navarra Hospital ComplexIdisna, Gastroenterology and Hepatology, Pamplona, Spain; ${ }^{5}$ Navarra Hospital Complex, Infectious Disease, Pamplona, Spain

\subsection{6/ejhpharm-2019-eahpconf.237}

Background The availability of new pangenotypic direct-acting antiviral (DAA) combinations has simplified the treatment of chronic hepatitis C. Clinical trials have shown high rates of sustained virological response (SVR), but there is a paucity of data in a real-life context.

Purpose To assess the effectiveness of glecaprevir/pibrentasvir (GLE/PIB), a pangenotypic DAA combination, for the treatment of hepatitis $\mathrm{C}$ virus (HCV) infection.

Material and methods A retrospective observational study for patients treated with GLE/PIB between November 2017 and April 2018 in a reference hospital. Variables analysed: sex, age, genotype, previous HCV therapy, HIV co-infection, METAVIR score (F0-F4) and DAA treatment duration. Effectiveness was evaluated as SVR12, defined as HCV-RNA titres $<15 \mathrm{IU} / \mathrm{mL} 12$ weeks after the end of treatment (post12). Data were collected from medical records and the database of drug dispensation by hospital pharmacists.

Results One-hundred and one patients were included (59\% men). Median age was 51 years (22-74). HCV genotypes: 30\% G1a; 19\% G1b; 1\% G1 no-subtyped; 4\% G2; and 28\% G3 and 18\% G4. Eleven patients had failed prior treatment (10 with interferon therapy and one with sofosbuvir/ ledipasvir). Twenty-six per cent of patients were $\mathrm{HIV} \mathrm{co}^{-}$ infected. Fibrosis grade was 12\% F4; 10\% F3; 20\% F2; and $58 \%$ F0-F1. Patients were treated for 8 weeks $(\mathrm{n}=88)$ or 12 weeks $(n=13)$. At the end of treatment one patient had positive viral load (VL) (G3, naïve, F2, monoinfected, 8 weeks of treatment). At post12, data on VL was available in 91 patients. Eighty-nine patients have eliminated $\mathrm{HCV}$ infection and two rebounded (G3, naïve, F0, monoinfected, 8 weeks of treatment and G2, naïve, F0, co-infected, 8 weeks of treatment). Ten patients had not yet had their VL analysed (three were lost to follow-up and seven will be available soon). Per protocol analysis, the rate of SVR was 97\% (95\% CI, 94 to 100 ), $97 \%$ in monoinfected vs $96 \%$ in co-infected patients. The most common adverse events were fatigue and headache, although treatment was well tolerated $(85 \%$ any adverse event).

Conclusion The combination GLE/PIB was effective with a high SVR12 rate, 97\%. Co-infected and monoinfected patients had a similar response with an optimal safety profile.

\section{REFERENCES AND/OR ACKNOWLEDGEMENTS}

EIPT-VHC project funded by the Spanish Ministry of Health and Carlos III Institute of Health.

No conflict of interest. 